\title{
A Note on Compactness of Commutators for Fractional Integrals Associated with Nondoubling Measures
}

\author{
J. J. Betancor and J. C. Fariña
}

\begin{abstract}
In this note we study the compactness of the commutator of fractional integrals associated with nondoubling measures.
\end{abstract}

Keywords. Commutators, fractional integral, compactness

Mathematics Subject Classification (2000). Primary 42B20, secondary 42B25

\section{Introduction}

In this note, given a positive Radon measure $\mu$ on $\mathbb{R}^{d}$ which may be nondoubling, we consider, for every $n \in \mathbb{N}$ and $0<\alpha<n \leq d$, the fractional integral $I_{\alpha}$ defined by

$$
I_{\alpha} f(x)=\int_{\mathbb{R}^{d}} \frac{f(y)}{|x-y|^{n-\alpha}} d \mu(y),
$$

for a suitable measurable function $f$ on $\mathbb{R}^{d}$. Our objective is to establish the compactness of the commutator operator of $I_{\alpha},\left[b, I_{\alpha}\right]$, given by

$$
\left[b, I_{\alpha}\right] f=b I_{\alpha} f-I_{\alpha}(b f),
$$

when $b$ belongs to certain subspace of the Tolsa's BMO type space ([30]) and $\mu$ satisfies the following growth property: there exists $C>0$ such that

$$
\mu(B(x, r)) \leq C r^{n}
$$

for every $x \in \mathbb{R}^{d}$ and $r>0, B(x, r)$ being the euclidean ball with center $x$ and radius $r$.

Departamento de Análisis Matemático, Universidad de La Laguna, 38271-La laguna, Tenerife, Islas Canarias, Spain; jbetanco@ull.es \& jcfarina@ull.es

Supported in part by grants from Ministerio de Ciencia y Tecnologa number MTM 2004-05878 and Consejera de Educacion Cultura y Deportes del Gobierno de Canarias, PI 2003/068. 
As it is wellknown, the paper of Coifman, Rochberg and Weiss [10] has played a central role in the investigations about commutators for integral operators. They characterizate the functions in the John and Nirenberg space $B M O\left(\mathbb{R}^{d}\right)$ like those functions $b$ for which the commutator operator $[b, T] f=$ $b(T f)-T(b f)$ is bounded from $L^{p}\left(\mathbb{R}^{d}\right)$ into itself for every Calderón-Zygmund operator T. In [10] two different proofs are presented for this result. One of them consists in proving a good $\lambda$ inequality. The other one uses complex analysis. Later, Stromberg (see [18]) gave a third proof of the commutator theorem by proving a pointwise estimate for a sharp maximal function. The Coifman-Rochberg-Weiss theorem has been extended for other operators and other spaces ([3, 7, 25, 26, 29] amongst others) following some of the three mentioned procedures.

Chanillo [7] investigated the boundedness in Lebesgue spaces or the commutator $\left[b, I_{\alpha}\right]$ when $\mu$ is the Lebesgue measure on $\mathbb{R}^{d}$. His results was completed and extended to Lipschitz spaces by Paluszynski [26].

If $X$ is a set, a quasidistance on $X$ is a function $d: X \times X \mapsto[0, \infty)$, such that

1. $d(x, y)=0$ if and only if $x=y$

2. $d(x, y)=d(y, x)$, for every $x, y \in X$

3. $d(x, y) \leq C_{d}(d(x, z)+d(z, y))$, for some $C_{d} \geq 1$ and for every $x, y, z \in X$.

Let $(X, d)$ be a set $X$ endowed with a quasidistance $d$ such that the balls associated with $d$ are open sets in the $d$-topology. Assume that $\mu$ is a positive Borel measure defined on a $\sigma$-algebra of subsets of $X$ which contains the $d$-balls, for which there exists a constant $C \geq 1$ such that the following doubling condition is satisfied:

$$
0<\mu\left(B_{d}(x, 2 r)\right) \leq C \mu\left(B_{d}(x, r)\right), \quad x \in X \text { and } r>0 .
$$

Then $(X, d, \mu)$ is called a homogeneous space.

Function spaces and operators on homogeneous spaces have been investigates by several authors (see, for instance, $[6,9,11,12,19,20,34]$ ). Fractional integrals on spaces of homogeneus type were studied by Gatto and Vagi [16], Gatto, Segovia and Vagi [15] and Sawyer and Wheeden [28]. More recently, Bramanti [3], Bramanti and Cerutti $([5,4])$ and Betancor [1] have extended the commutator theorems of Coifman, Rochberg and Weiss and Chanillo to the setting of homogeneous spaces.

Last years central results of classical Calderón-Zygmund theory have been established when the doubling condition (1) on the underlying measure is not satisfied. In this case $(X, d, \mu)$ is called a non homogeneous space. Tolsa [30, 31, $32]$ and, independently, Nazarov, Treil and Volberg [22, 23, 24] have shown that the doubling condition (1) was not really necessary. In the survey of Verdera [35] 
the interested reader can find a nice exposition of results on Calderón-Zygmund theory on nonhomogeneous spaces.

Tolsa [30] introduced a good substitute in the nondoubling case for the space $B M O$. He denoted it by $R B M O(\mu)$. Given a cube $Q \subset \mathbb{R}^{d}$ with sides parallel to the axes, we choose $N$ as the smallest nonnegative integer such that $2^{N} Q$ is doubling, that is, $\mu\left(2^{N+1} Q\right) \leq \beta_{d} \mu\left(2^{N} Q\right)$, where $\beta_{d}$ is defined in [30, Remark 2]. By $\tilde{Q}$ we denote the cube $2^{N} Q$. Let $Q \subset R$ be two cubes in $\mathbb{R}^{n}$. By $N_{Q, R}$ we represent the first integer $k$ for which $l\left(2^{k} Q\right) \geq l(R)$ (in case $\mathbb{R}^{d}=R \neq Q$ we set $\left.N_{Q, R}=\infty\right)$. Here $l(R)$ denotes the length of the side of $R$. We set

$$
K_{Q, R}=1+\sum_{k=1}^{N_{Q, R}} \frac{\mu\left(2^{k} Q\right)}{l\left(2^{k} Q\right)^{n}} .
$$

Let $\rho>1$. We say that $f \in L_{l o c}^{1}(\mu)$ is in $R B M O(\mu)$ if there exists $C>0$ such that for every cube $Q$ (centered at some point of $\operatorname{supp}(\mu))$

$$
\frac{1}{\mu(\rho Q)} \int_{Q}\left|f-m_{\tilde{Q}} f\right| d \mu \leq C,
$$

and $\left|m_{Q} f-m_{R} f\right| \leq C K_{Q, R}$ for any two doubling cubes $Q \subset R$. The minimal constant $C$ satisfying the above two conditions is denoted by $\|f\|_{*}$. Thus $\|\cdot\|_{*}$ is a norm in $R B M O(\mu)$ modulus additive constants. The space $R B M O(\mu)$ is not depending on $\rho$.

The space $R B M O(\mu)$ is suitable for the nondoubling measures because it has a lot of properties of the ones of the classical space $B M O$, including some properties that were not satisfied by the spaces in $[21,24]$. In [30], the predual of $R B M O(\mu)$ is described as the atomic space $H_{a t b}^{1, \infty}(\mu)$. If $T$ is a CalderónZygmund operator bounded from $L_{2}(\mu)$ into itself, then it is also bounded from $H_{\text {atb }}^{1, \infty}(\mu)$ into $L_{1}(\mu)([30$, Theorem 8.1]). By using a sharp maximal operator in [30, Theorem 9.1] it is proved that the commutator $[b, T]$ is bounded in $L_{p}(\mu)$, $1<p<\infty$, for every Calderón-Zygmund operator $T$ bounded in $L_{2}(\mu)$, provided that $b \in R B M O(\mu)$. Gatto and García Cuerva [13, 14] have investigated the fractional integral on nonhomogeneous spaces in Lebesgue and Lipschitz spaces. Recently the ideas contained in the proof of the [30, Theorem 9.1] were used by Chen and Sawyer in [8], where they obtain the commutator theorem for the fractional integrals $I_{\alpha}$ associated with a nondoubling measures in $\mathbb{R}^{d}$. Also $\mathrm{Hu}$, Meng and Yang [17] have obtained the $\left(L^{p}, L^{q}\right)$-boundedness and the weak type end point estimate for the multilinear commutator by fractional integrals with $R B M O(\mu)$ functions. By using an uniform boundedness property for the fractional integrals $I_{\alpha}$ (Lemma 2.2) we prove the compactness of the commutator operator $\left[b, I_{\alpha}\right]$ when $b \in R C M O(\mu)$, where like in [33], $R C M O(\mu)$ denotes the closure of the space $\mathcal{D}\left(\mathbb{R}^{d}\right)$, of the smooth and compact supported functions 
on $\mathbb{R}^{d}$, in $R B M O(\mu)$. Note that $C M O$-type spaces considered by Uchiyama [33] does not coincide with the $V M O$ space introduced by Sarason [27] (also see [2]). However Coifman and Weis [12, p. 638] called VMO to a space of $C M O$-type. Our Theorem 3.1 can be seen as an extension to nondoubling setting for $I_{\alpha}$ of [33, Theorem 2] for Calderón-Zygmund operators.

Throughout this paper, by $C$ we always will denote a positive constant that can be changed from one line to the other line.

\section{The compactness of the commutator for fractional integrals}

Our objective in this section is to see that if $b \in R C M O(\mu)$ then the commutator operator $\left[b, I_{\alpha}\right]$ is compact from $L_{p}(\mu)$ into $L_{q}(\mu)$ provided that $1<p<\frac{n}{\alpha}$ and $\frac{1}{q}=\frac{1}{p}-\frac{\alpha}{n}$. Our result can be seen as an extension for the fractional integral $I_{\alpha}$ associated with nondoubling measures on $\mathbb{R}^{d}$ of the result due to Uchiyama (see [33, Theorem 2]) for Calderón-Zygmund singular integral operators.

We need, before establishing our compactness result, to prove two lemmas that will be useful in the sequel. In the first one we state a compactness criterion in $L_{p}(\mu)$ that is a modification of [36, Theorem, p. 275].

Lemma 2.1. Let $B$ a subset of $L_{p}(\mu)$ where $1 \leq p<\infty$. Then, $B$ is relatively compact in $L_{p}(\mu)$ provided that the following properties are satisfied:

i) $\sup _{f \in B}\|f\|_{p}<\infty$;

ii) the mapping $\begin{aligned} \mathbb{R}^{d} & \mapsto L_{p}(\mu) \\ x & \mapsto f(\cdot+x)\end{aligned}$ is equicontinuous in $B$;

iii) $\lim _{m \rightarrow \infty} \int_{|x| \geq m}|f(x)|^{p} d \mu(x)=0$, uniformly in $B$.

Proof. This lemma can be proved, with minor changes, by proceeding as in [36, p. 275-276].

Lemma 2.2. Let $1<p<\frac{n}{\alpha}, \frac{1}{q}=\frac{1}{p}-\frac{\alpha}{n}$ and $0<\alpha<n$. Then there exists $C>0$ such that

$$
\left\|I_{\alpha}(f)(x+\cdot)\right\|_{q} \leq C\|f\|_{p}, \quad f \in L_{p}(\mu) \text { and } x \in \mathbb{R}^{d}
$$

Proof. To see this property we study carefully the proof of [13, Theorem 3.2]. We are going to prove that there exists $C>0$ such that, for $f \in L_{p}(\mu)$ and $x \in \mathbb{R}^{d}$,

$$
\mu\left\{y \in \mathbb{R}^{d}:\left|I_{\alpha} f(x+y)\right|>\lambda\right\} \leq C\left(\frac{\|f\|_{p}}{\lambda}\right)^{q}, \quad \lambda>0 .
$$

Here $C$ is not depending on $x \in \mathbb{R}^{d}$. 
Without lost of generality we assume that $f \geq 0$ and $\|f\|_{p}=1$. Let $\lambda>0$. We can write

$$
\begin{aligned}
I_{\alpha} f(x+y) & =\int_{B(x+y, r)} \frac{f(z)}{|x+y-z|^{n-\alpha}} d \mu(z)+\int_{\mathbb{R}^{d} \backslash B(x+y, r)} \frac{f(z)}{|x+y-z|^{n-\alpha}} d \mu(z) \\
& =: I+I I
\end{aligned}
$$

$r>0$ and $x, y \in \mathbb{R}^{d}$. Hölder's inequality implies that

$$
|I I| \leq\left(\int_{\mathbb{R}^{d} \backslash B((x+y, r)} \frac{1}{|x+y-z|^{(n-\alpha) p^{\prime}}} d \mu(z)\right)^{\frac{1}{p^{\prime}}} .
$$

By using [13, Lemma 2.2] we get

$$
\int_{\mathbb{R}^{d} \backslash B((x+y, r)} \frac{1}{|x+y-z|^{(n-\alpha) p^{\prime}}} d \mu(z) \leq C r^{-\gamma}, \quad r>0, x, y \in \mathbb{R}^{d}
$$

where $\gamma=n\left(p^{\prime}-1\right)-\alpha p^{\prime}$. Then $|I I| \leq C r^{-\left(\frac{n}{p}-\alpha\right)}, r>0, x, y \in \mathbb{R}^{d}$. Assume that $C r^{-\left(\frac{n}{p}-\alpha\right)}=\frac{\lambda}{2}$. Thus we have that $\left\{y \in \mathbb{R}^{d}:\left|I_{\alpha}(f)(x+y)\right|>\lambda\right\} \subset\{y \in$ $\left.\mathbb{R}^{d}:|I|>\frac{\lambda}{2}\right\}, x \in \mathbb{R}^{d}$. Moreover, by [13, Lemma 2.1],

$$
\begin{aligned}
|I| & \leq\left(\int_{B(x+y, r)} \frac{|f(z)|^{p}}{|x+y-z|^{n-\alpha}} d \mu(z)\right)^{\frac{1}{p}}\left(\int_{B(x+y, r)} \frac{d \mu(z)}{|x+y-z|^{n-\alpha}}\right)^{\frac{1}{p^{\prime}}} \\
& \leq C r^{\frac{\alpha}{p^{\prime}}}\left(\int_{B(x+y, r)} \frac{|f(z)|^{p}}{|x+y-z|^{n-\alpha}} d \mu(z)\right)^{\frac{1}{p}}, \quad x, y \in \mathbb{R}^{d} .
\end{aligned}
$$

Hence, Chebyshev's inequality leads to

$$
\begin{aligned}
\mu\left\{y \in \mathbb{R}^{d}:\left|I_{\alpha}(f)(x+y)\right|>\lambda\right\} & \leq C \frac{r^{\frac{\alpha p}{p^{\prime}}}}{\lambda^{p}} \int_{\mathbb{R}^{d}} \int_{B(x+y, r)} \frac{|f(z)|^{p}}{|x+y-z|^{n-\alpha}} d \mu(z) d \mu(y) \\
& =C \frac{r^{\frac{\alpha p}{p^{\prime}}}}{\lambda^{p}} \int_{\mathbb{R}^{d}} \int_{B(z-x, r)} \frac{d \mu(y)}{|z-x-y|^{n-\alpha}}|f(z)|^{p} d \mu(z) \\
& \leq C \lambda^{-q}, \quad x \in \mathbb{R}^{d} .
\end{aligned}
$$

Thus (2) is established.

To finish the proof of our lemma it is sufficient to use Marcinkiewicz's interpolation theorem.

We now establish our compactness result for the commutator associated to the fractional integral $I_{\alpha}$.

Theorem 2.3. Let $1<p<\frac{n}{\alpha}, \frac{1}{q}=\frac{1}{p}-\frac{\alpha}{n}$ and $0<\alpha<n$. Assume that $b \in R C M O(\mu)$. Then the commutator operator $\left[b, I_{\alpha}\right]$ is compact from $L_{p}(\mu)$ into $L_{q}(\mu)$. 
Proof. Since $b \in R C M O(\mu), b$ is in $R B M O(\mu)$ and, according to [8, Theorem 1], the commutator operator $\left[b, I_{\alpha}\right]$ is bounded from $L_{p}(\mu)$ into $L_{q}(\mu)$. Moreover, there exist $C>0$ such that

$$
\left\|\left[b, I_{\alpha}\right] f\right\|_{q} \leq C\|b\|_{*}\|f\|_{p}, \quad f \in L_{p}(\mu),
$$

where $C$ does not depend on $b$. By (3), we can assume that $b \in \mathcal{D}\left(\mathbb{R}^{d}\right)$.

Our objective is to show that the subset

$$
B=\left\{\left[b, I_{\alpha}\right] f:\|f\|_{p} \leq 1\right\}
$$

of $L_{q}(\mu)$ satisfies the condition (i), (ii) and (iii) in Lemma 2.1 when $p$ is replaced by $q$. From (3) we immediately deduce that $\sup _{\|f\|_{p} \leq 1}\left\|\left[b, I_{\alpha}\right] f\right\| \leq C\|b\|_{*}$. Hence (i) in Lemma 2.1 is satisfied by $B$.

For every $x, z_{1}, z_{2} \in \mathbb{R}^{d}$, we can write

$$
\begin{aligned}
{\left[I_{\alpha}, b\right] f } & \left(x+z_{1}\right)-\left[I_{\alpha}, b\right] f\left(x+z_{2}\right) \\
= & \int_{\mathbb{R}^{d}}\left(\frac{1}{\left|x+z_{1}-y\right|^{n-\alpha}}-\frac{1}{\left|x+z_{2}-y\right|^{n-\alpha}}\right)\left(b(y)-b\left(x+z_{2}\right)\right) f(y) d \mu(y) \\
& +\int_{\mathbb{R}^{d}} \frac{1}{\left|x+z_{1}-y\right|^{n-\alpha}}\left(b\left(x+z_{2}\right)-b\left(x+z_{1}\right)\right) f(y) d \mu(y), \quad f \in L_{p}(\mu) .
\end{aligned}
$$

Hence, for every $x, z_{1}, z_{2} \in \mathbb{R}^{d}$, we get

$$
\begin{aligned}
\mid\left[I_{\alpha}, b\right] & f\left(x+z_{1}\right)-\left[I_{\alpha}, b\right] f\left(x+z_{2}\right) \mid \\
\leq & \int_{\left|x-y+z_{1}\right|>\left|z_{1}-z_{2}\right|} \frac{\left|b\left(x+z_{1}\right)-b\left(x+z_{2}\right)\right|}{\left|x+z_{1}-y\right|^{n-\alpha}}|f(y)| d \mu(y) \\
& +\int_{\left|x-y+z_{1}\right|>\left|z_{1}-z_{2}\right|}\left|\frac{1}{\left|x+z_{1}-y\right|^{n-\alpha}}-\frac{1}{\left|x+z_{2}-y\right|^{n-\alpha} \mid}\right| \\
& \cdot\left|b(y)-b\left(x+z_{2}\right)\right||f(y)| d \mu(y) \\
& +\int_{\left|x-y+z_{1}\right| \leq\left|z_{1}-z_{2}\right|} \frac{1}{\left|x+z_{1}-y\right|^{n-\alpha}}\left|b(y)-b\left(x+z_{1}\right)\right||f(y)| d \mu(y) \\
& +\int_{\left|x-y+z_{1}\right| \leq\left|z_{1}-z_{2}\right|} \frac{1}{\left|x+z_{2}-y\right|^{n-\alpha}}\left|b(y)-b\left(x+z_{2}\right)\right||f(y)| d \mu(y) \\
\leq & C\left|z_{1}-z_{2}\right|\left(I_{\alpha}(|f|)\left(x+z_{1}\right)+\int_{\left|x-y+z_{1}\right|>\left|z_{1}-z_{2}\right|}\left|x+z_{2}-y\right|^{n-\alpha}|f(y)| d \mu(y)\right. \\
& +\int_{\left|x-y+z_{1}\right| \leq\left|z_{1}-z_{2}\right|} \frac{1}{\left|x+z_{1}-y\right|^{n-\alpha}}|f(y)| d \mu(y) \\
& \left.+\int_{\left|x-y+z_{1}\right| \leq\left|z_{1}-z_{2}\right|} \frac{1}{\left|x+z_{2}-y\right|^{n-\alpha}}|f(y)| d \mu(y)\right) \\
\leq & C\left|z_{1}-z_{2}\right|\left(I_{\alpha}(|f|)\left(x+z_{1}\right)+I_{\alpha}(|f|)\left(x+z_{2}\right)\right), \quad f \in L_{p}(\mu) .
\end{aligned}
$$


By invoking now Lemma 2.2, we conclude that, for every $z_{1}, z_{2} \in \mathbb{R}^{d}$,

$\left\|\left[I_{\alpha}, b\right](f)\left(\cdot+z_{1}\right)-\left[I_{\alpha}, b\right](f)\left(\cdot+z_{2}\right)\right\|_{q} \leq C\left|z_{1}-z_{2}\right|\|f\|_{p} \leq C\left|z_{1}-z_{2}\right|, \quad\|f\|_{p} \leq 1$.

Thus (ii) in Lemma 2.1 is verified by $B$.

On the other hand, since $b \in \mathcal{D}\left(\mathbb{R}^{d}\right)$, when $|x|$ is sufficiently large we have

$$
\left|\left[I_{\alpha}, b\right] f(x)\right| \leq \int_{\mathbb{R}^{d}} \frac{|b(y)||f(y)|}{|x-y|^{n-\alpha}} d \mu(y) \leq \frac{C}{|x|^{n-\alpha}}\|f\|_{p} .
$$

Then, if $m$ is large enough,

$$
\int_{|x| \geq m}\left|\left[I_{\alpha}, b\right] f(x)\right|^{q} d \mu(x) \leq C\|f\|_{p} \int_{|x| \geq m}|x|^{-(n-\alpha) q} d \mu(x) .
$$

Hence by using [13, Lemma 2.2], since $(n-\alpha) q-n>0$, we conclude that

$$
\int_{|x| \geq m}\left|\left[I_{\alpha}, b\right] f(x)\right|^{q} d \mu(x) \leq C m^{n-(n-\alpha) q}\|f\|_{q} \rightarrow 0, \quad \text { as } m \rightarrow \infty,
$$

and (iii) in Lemma 2.1 is established for $B$. Thus the proof is completed.

\section{References}

[1] Betancor, J. J., A commutator theorem for fractional integrals in spaces of homogeneous type. Int. J. Math. Math. Sci. 24 (2000)(6), $403-418$.

[2] Bourdaud, G., Lanza de Cristoforis, M. and Sickel, W., Functional calculus on BMO and related spaces. J. Funct. Anal. 189 (2002)(2), 515 - 538.

[3] Bramanti, M., Commutators of integral operators with positive kernels. Le Matematiche 49 (1994)(1), 149 - 168.

[4] Bramanti, M. and Cerutti, M. C., Commutators of singular integrals and fractional integrals on homogeneous spaces. In: Harmonic Analysis and Operator Theory (Caracas 1994), Contemp. Math. 189. Providence, RI: Amer. Math. Soc. 1995, pp. $81-94$.

[5] Bramanti, M. and Cerutti, M. C., Commutators of singular integrals on homogeneous spaces. Boll. Un. Mat. Ital. B (7) 10 (1996) (4), 843 - 883.

[6] Burger, N., Espace de fonctions à variation moyenne bornée sur un espace de nature homogène. C. R. Acad. Sc. Paris 286 (1978), 139 - 142.

[7] Chanillo, S., A note on commutators. Indiana Univ. Math. J. 31 (1982)(1), $7-16$.

[8] Chen, W. and Sawyer, E., A note on commutators of fractional integrals with RBMO( $\mu$ ) functions. Illinois Math. J. 46 (2002)(4), $1287-1298$. 
[9] Coifman, R. and de Guzmán, M., Singular integrals and multipliers on homogeneous spaces. Rev. Un. Mat. Argentina 25 (1970), 137 - 143.

[10] Coifman, R. R., Rochberg, R. and Weiss, G., Factorization theorems for Hardy spaces in several variables. Ann. Math. 103 (1976), 611 - 635.

[11] Coifman, R. R. and Weiss, G., Analyse Harmonique Non-Commutative sur Certain Espaces Homogènes. Lectures Notes Math. 242, Berlin: Springer 1971.

[12] Coifman, R. R. and Weiss, G., Extension of Hardy spaces and their use in analysis. Bull. Amer. Math. Soc. 83 (1977)(4), $569-645$.

[13] García-Cuerva, J. and Gatto, A. E., Boundedness properties of fractional integral operators associated to non-doubling measures. Studia Math. 162 (2004)(3), $245-261$.

[14] García-Cuerva, J. and Gatto, A. E., Lipschitz spaces and Calderón-Zygmund operators associated to nondoubling measures. Publ. Mat. 49 (2005)(2), $285-296$.

[15] Gatto, A. E., Segovia, C. and Vági, S., On fractional differentation and integration on spaces of homogeneous type. Rev. Mat. Iberoamericana 12 (1996)(1), $111-145$.

[16] Gatto, A. E. and Vagi, S., Fractional Integral on Spaces of Homogeneous Type. Analysis and Partial Differential Equations. Lectures Notes Pure Appl. Math. 122. New York: Dekker 1990, pp. $171-216$.

[17] Hu, G., Meng, Y. and Yang, D., Multilinear commutators for fractional integrals in non-homogeneous spaces. Publ. Mat. 48 (2004), 335 - 367.

[18] Janson, S., Mean oscillation and commutators of singular integral operators. Ark. Math. 16 (1978), 263 - 270.

[19] Macías, R. A. and Segovia, C., Lipschitz functions on spaces of homogeneous type. Adv. Math. 33 (1979)(3), 257 - 270.

[20] Macías, R. A. and Segovia, C., A decomposition into atoms of distributions on spaces of homogeneous type. Adv. Math. 33 (1979)(3), $257-270$.

[21] Mateu, J., Mattila, P., Nicolau, A. and Orobitg, J., BMO for non doubling measures. Duke Math. J. 102 (2000)(3), 533 - 565.

[22] Nazarov, F. L., Treil, S. R. and Volverg, A. L., Weak type estimates and Cotlar inequalities for Calderón-Zygmund operators on nonhomogeneous spaces. Internat. Math. Res. Notices 9 (1998), 463 - 487.

[23] Nazarov, F. L., Treil, S. R. and Volverg, A. L., Accretive system Tb-theorems on nonhomogeneous spaces. Duke Math. J. 113 (2002)(2), 259 - 312.

[24] Nazarov, F. L., Treil, S. R. and Volverg, A. L., The Tb-theorem on nonhomogeneous spaces. Acta Math. 190 (2003)(2), $151-239$.

[25] Orobitg, J. and Pérez, C., $A_{p}$ weights for nondoubling measures in $\mathbb{R}^{d}$ and applications. Trans. Amer. Math. Soc. 354 (2002)(5), $2013-2033$.

[26] Paluszyński, M., Characterization of the Besov spaces via the commutator operator of Coifman, Rochberg and Weiss. Indiana Univ. Math. J. 44 (1995)(1), $1-17$. 
[27] Sarason, D., Functions of vanishing mean oscillation. Trans. Amer. Math. Soc. 207 (1975), $391-405$

[28] Sawyer, E. and Wheeden, R. L., Weighted inequalities for fractional integrals on Euclidean and homogeneous spaces. Amer. J. Math. 114 (1992), 813 - 874.

[29] Segovia, C. and Torrea, J. L., Higher order commutators for vector valued Calderón-Aygmund operators. Trans. Amer. Math. Soc. 336 (1993), 537 - 556.

[30] Tolsa, X., BMO, H1 and Calderón-Zygmund operators for noo doubling measures. Math. Annalen 319 (2001), 89 - 149.

[31] Tolsa, X., L2-boundedness of the Cauchy integral operators for continuous measures. Duke Math. J. 98:2 (1999), 269 - 304.

[32] Tolsa, X., A T(1) theorem for nondoubling measures with atoms. Proc. London Math. Soc. 82 (2001)(3), $195-228$.

[33] Uchiyama, A., On the compactness of operators of Hankel type. Tohoku Math. J. 30 (1978), 163 - 171.

[34] Uchiyama, A., The factorization of $H^{p}$ on the space of homogeneous type. Pacific J. Math. 92 (1981)(2), 453 - 468.

[35] Verdera, J., The fall of the doubling condition in Calderón-Zygmund theory. Publ. Mat. (2002), 275 - 292.

[36] Yosida, K., Functional Analysis. Berlin: Springer 1968.

Received June 24, 2005 